\title{
Why the Glove of Mathematics Fits the Hand of the Natural Sciences So Well : How Far Down the (Fibonacci) Rabbit Hole Goes
}

\author{
David F. Haight \\ Department of History and Philosophy, Plymouth State University \\ Plymouth, New Hampshire, U.S.A.
}

doi: 10.19044/esj.2016.v12n15p1 URL:http://dx.doi.org/10.19044/esj.2016.v12n15p1

\begin{abstract}
Why does the glove of mathematics fit the hand of the natural sciences so well? Is there a good reason for the good fit? Does it have anything to do with the mystery number of physics or the Fibonacci sequence and the golden proportion? Is there a connection between this mystery (golden) number and Leibniz's general question, why is there something (one) rather than nothing (zero)? The acclaimed mathematician G.H. Hardy (1877-1947) once observed: "In great mathematics there is a very high degree of unexpectedness, combined with inevitability and economy." Is this also true of great physics? If so, is there a simple "preestablished harmony" or linchpin between their respective ultimate foundations? The philosopher-mathematician, Gottfried Leibniz, who coined this phrase, believed that he had found that common foundation in calculus, a methodology he independently discovered along with Isaac Newton. But what is the source of the harmonic series of the natural log that is the basis of calculus and also Bernhard Riemann's harmonic zeta function for prime numbers? On the occasion of the three-hundredth anniversary of Leibniz's death and the one hundredth-fiftieth anniversary of the death of Bernhard Riemann, this essay is a tribute to Leibniz's quest and questions in view of subsequent discoveries in mathematics and physics. (In the Journal of Interdisciplinary Mathematics, Dec. 2008 and Oct. 2010, I have already sympathetically discussed in detail Riemann's hypothesis and the zeta function in relation to primes and the zeta zeros. Both papers were republished online in 2013 by Taylor and Francis Scientific Publishers Group.)
\end{abstract}

Keywords: Fibonacci sequence, the "prime" prime number, the fine structure constant of hydrogen, Planck's constant, other physical constants, the principle of indeterminacy, the pre-established harmony, the harmonic 
series, the natural log, the binary code, and the digital code

\section{Introduction}

Mathematics is the science of the most complete abstractions to which the human mind can attain. As such, it has provoked and inspired more philosophical and scientific questions about its foundations and its relation to the world than any other scientific discipline. The abstractions known as numbers are the backbone of science. If a subject cannot be described by numbers, it is not scientific. What, however, are these abstract entities known as numbers and why do they relate so well to the concrete physical sciences? Einstein's felicitous use of Bernhard Riemann's fourdimensional curved geometry and metric sphere to mathematically model his general theory of relativity is a stunning example of this enigmatic relationship between hand and glove. The glove of abstract mathematics existed for over half a century before the physical hand of the universe put it on, so to speak. Was mathematics also ready-made for quantum physics?

Both the fine structure constant of hydrogen $(1 / 137+)$ - the prime (first) element - and the 1/137+ probability of the electron jump are derived from Schrödinger's sovereign wave equation, which is itself derived from Euler's and Fourier's unique exponential growth function that nature had been wearing long before Euler and Fourier discovered it (and Schrödinger rediscovered it). In recent years, Leibniz's and Euler's exponential growth (catenary) curve has also turned up in foundational cosmology in mathematically modeling the inflationary growth of the newborn universe. Even the so-called "imaginary" number, the square root of minus one, has turned out to be "real." Vital and fundamental to both relativity theory and quantum mechanics, this unusual number is also essential to the dimensionless origin of dimensions and is equal to the speed of light for reasons that we will mention.

All of these examples, plus many more, strikingly attest to the prescient nature of mathematics and what the philosopher-mathematician Gottfried Leibniz, co-founder of calculus, referred to as the "pre-established harmony" between mind and nature [Robert Latta, 1965]. He based this idea on the exponential growth function (necklace), $y=e^{x}=$ the harmonic power series, which is surprisingly unique in that it is the only exponential function (along with its constant multiples) that is its own derivative. This means that its state of growth is the same as its rate of growth-they are clasp together in a unique harmony. As we will see, this function that is unlike any other gives us a big clue as to the fundamental symmetry or "pre-established harmony" of mathematics and the physical sciences.

That there is such a fundamental connection between physics and mathematics is strongly supported in part by the fact that when 
any two numbers are added together and then the latter number is divided by the former, the resulting numbers inevitably converge to a number very close to the fine structure constant of hydrogen, 137+. That number is the 137.5degree smaller segment of a circle divided according to the classic golden proportion, which is unlike any other proportion because it is selfreferentially self-derived. Significantly, this golden ratio number also determines the possible histories of the electron of the hydrogen atom; the limit of the sum over the possible histories of the hydrogen electron is the golden proportion [H. E. Huntley, 1970]. Is the connection in hydrogen between the golden ratio and its fine structure constant, 137+, in quantum mechanics just a coincidence, or does this connection go to the heart of both mathematics and the physical sciences? Because there is a necessary connection between relativity theory and quantum mechanics by means of the special number $137+$-according to the astounding formula, alpha $\left(\alpha^{-1}\right)$ equals $h c / 2$ pi $e^{2}$ equals $137+$, where $h$ stands for Planck's quantum constant of action, $c$ stands for Einstein's constant of light, and $e$ stands for the charge of the electron (and because of Rydberg's constant for atomic shells which contains the number $137+$ ) - I am going to maintain that there is a necessary connection. Not only do any two numbers when collected (integrated or spliced) and divided (differentiated or sliced), as in calculus, converge to the golden ratio known as Phi (because they are so close together, the numbers in the Fibonacci sequence converge to Phi faster than any other two numbers in a sequence), but all of the fundamental operations and operators of mathematics appear in Leonhard Euler's spectacular identity, which physicist Richard Feynman referred to as "the most remarkable formula in mathematics" [Paul J. Nahim, 1998.]: $e^{\pi i}$ equals -1 . As Feynman himself discovered, when Phi is inserted into it, Euler's stunning formula becomes $e$ to the power of Pi times $i=1 / \mathrm{Phi}-\mathrm{Phi}$. Correspondingly, the natural $\log e=$ $1.37^{\mathrm{Pi}}$, $^{\mathrm{Phi}^{1.37}}=\mathrm{Pi} / \mathrm{Phi}$, and $13.7^{1.37} / \mathrm{Phi}^{2}=13.7$, making a strong connection between another set of three fundamental constants. Furthermore, Leibniz showed that all numbers can be translated into the binary scale of zeros and ones, and-what Leibniz did not know--the binary code is based on Phi's radical roots (the simplest nested radical) that oscillate back and forth between zero and one.

Leibniz rightly claimed "that all lines of motion in the whole of geometry are reduced to two motions only, one in a straight line and the other in a circle" [G. H. R. Parkinson, 1983], similar to the compression of all numbers to strings of zeros and ones. Beautifully, and amazingly, the line and the circle are the limiting cases of the golden Phi logarithmic spiral. A line is the minimum possible area of the spiral, and a circle is the maximum possible area; all other polygons are intermediate combinations between 
these two figures. What Leibniz did not discover in both cases is that Phi is the unique unity-in-diversity that bridges the gap between these binary opposites. A one-dimensional straight line can be transformed and moved into a two-dimensional circle because of the joint arithmetic and geometric character of non-dual Phi. This (golden) intersection between dimensions is the result of the unique intersection of the dimensionless golden section with itself where infinity comes to rest.

Leibniz's particular version of the law of continuity between dimensions (derivatives) and the corresponding relationship between the limit and the unlimited in a mathematical progression also depend upon Phi, since by multiplication and addition to a line a plane is produced; by multiplication and addition to a plane a solid is produced; etc. In the golden logarithmic spiral, the intervals add up while the ratio of frequencies multiply; this means that the radial angle adds up in an arithmetic progression at the same time that the spiral radius grows up in a geometric expansion. Together these two progressions produce the harmonic progression of all the subsequently derived dimensions. These two progressions come right out of Descartes's equiangular spiral of analytic geometry, the “giant” inspiring (spiral) forerunner of Leibniz's and Newton's calculus. Phi is the only proportion that remains the same or true to itself by both adding and multiplying itself, which makes it the portal or "black (Fibonacci rabbit) hole” connecting one dimension to another, the common boundary/coupling constant between linear and non-linear expansion, order and chaos, the limit and the unlimited. In short, it is the binary switch between dimensions.

\section{(Natural) logging into the universe's digital computer: be wise--digitize}

Leibniz's translation of the decimal code into the binary system also shows a deep correlation between unity (ones) and the progression of $1,3,7$ that consistently appears whenever we apply the block (142857) and period (1, 3, 2, 6, 4, 5) of the special number seven, the smallest possible number that when divided into unity makes a period of recurring digits: 


\begin{tabular}{|c|c|c|c|c|c|c|}
\hline Decin & & Binary & & cimal & & Binary \\
\hline 1 & $=2^{0}$ & $=1=$ & $0 \times 7+1$ & 17 & $=$ & 10001 \\
\hline 2 & $=2^{1}$ & $=10=$ & $1 \times 7+3$ & 18 & $=$ & 10010 \\
\hline 3 & & $=11$ & & 19 & $=$ & 10011 \\
\hline 4 & $=2^{2}$ & $=100=$ & $14 \times 7+2$ & 20 & $=$ & 10100 \\
\hline 5 & & $=101$ & & 21 & $=$ & 10101 \\
\hline 6 & & $=110$ & & 22 & $=$ & 10110 \\
\hline 7 & & $=111$ & & 23 & $=$ & 10111 \\
\hline 8 & $=2^{3}$ & $=1000=$ & $=142 \times 7+6$ & 24 & $=$ & 11000 \\
\hline 9 & & $=1001$ & & 25 & $=$ & 11001 \\
\hline 10 & & $=1010$ & & 26 & $=$ & 11010 \\
\hline 11 & & $=1011$ & & 27 & $=$ & 11011 \\
\hline 12 & & $=1100$ & & 28 & $=$ & 11100 \\
\hline 13 & & $=1101$ & & 29 & $=$ & 11101 \\
\hline 14 & & $=1110$ & & 30 & $=$ & 11110 \\
\hline 15 & & $=1111$ & & 31 & $=$ & 11111 \\
\hline 16 & $=2^{4}$ & $=10000$ & $=1428 \times 7+4$ & $32=2^{5}$ & $=$ & $\begin{array}{c}100000=14285 \times 7+5 \\
\text { Etc. }\end{array}$ \\
\hline
\end{tabular}

Does the reader see how all the above strings of binary ones are produced because of the $1,3,7, \ldots$ progression? If not, count the numbers in between all of the binary numbers that have only ones in them. This 1, 3, 7, ... progression provides us with a deep clue as to the unity [ones] of the binary number code. As shown in the table above, here are the block and period of the prime number seven that generates this $1,3,7, \ldots$ sequence:

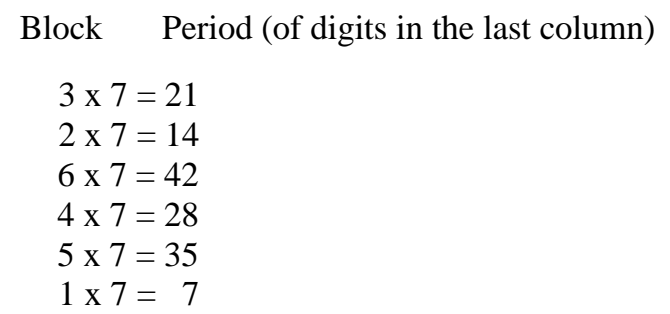

In brief, all numbers can be digitally reduced to sums of the powers of two, and all digital numbers can be compressed to a set of ones through the block and period of the number seven and the Phi number progression of $1,3,7, \ldots$ One number is all-all numbers are one.

Leibniz also knew that this same progression appears whenever solutions to the simple binary formula $2^{n}-1$ are found: $2^{0}-1=0,2^{1}-1=1$, $2^{2}-1=3,2^{3}-1=7$, etc.--all these numbers being one less than a power of two. (The number two and its powers appear more often in mathematics and physics than any other numbers.) This remarkable connection between the formula $2^{n}-1$ and the binary code bears further mention from an authoritative source: "Expressed as binary numbers, all numbers of the form $2^{n}-1$ consist exclusively of 1 's, namely, exactly $n 1$ 's. For example, $M_{4}=$ $2^{4}-1=15$, or 1111 in binary” [Manfred R. Schroeder, 1984]. 
This formula is, for Leibniz, one of the reasons why there is something rather than nothing, i.e., a reason for the origin of things. It's like the principle of least action-how easily and effortlessly something comes from nothing - that Leibniz also discovered: from zero to one and so on. The shortest "distance" between nothing and something (zero and one), which are the first two numbers of the Fibonacci number sequence, is the least amount of action (energy times time), like the pure dimensionless number 137+ of Planck's constant of action $h$. What Leibniz did not know is that the golden proportion, as mentioned above, is also the link between nothing and something in terms of the (quantum) jump from one dimension to another that thereby relates Phi to the quantum of stationary action (137+). Something is easier than nothing; a complete vacuum is difficult to create and sustain. "[The golden ratio] is a scale of proportions which makes the bad difficult and the good easy," said Einstein after receiving Le Corbusier's golden modular for designing architecture [Mario Livio, 2002].

There is quite stunningly yet another well-known connection between the formula $2^{n}-1$ and prime numbers, which are the so-called "building blocks" of mathematics: if and only if $2^{n}-1$ divides the $(n-1)$ th term of a series, then $2^{n}-1$ is prime; otherwise, it is composite. Almost magically, the Mersenne sequence of primes, according to $M p=2^{p}-1$, also produces the progression of $1,3,7$ that figures prominently in the binary and decimal number systems. Moreover, prime numbers, the "atoms" of mathematics, are necessarily related to the atoms of nature because of the well-known Rydberg rule that follows the same pattern as Euler's harmonic zeta power series (derived from the self-derived exponential growth function): Zeta $(x)=1 / 1^{x}+1 / 2^{x}+1 / 3^{x}+1 / 4^{x}+1 / 5^{x} \ldots=\mathrm{pi}^{2} / 6$, which is the inverse of the probability that any two numbers selected at random will be prime to one another.

The special number 137+ also appears in the Rydberg constant for hydrogen and sets the scale for the allowed energy levels or shells of the electron, and hence the subsequent development of the universe: $16 \pi \sqrt{ } 5$ (all divided by 3) $\mathrm{x} 136^{2} \times 137 \sigma$, or $R=1 / 2(1 / 137)^{2} \mu c / 2 \pi h$. (It is worth mentioning that Rydberg's constant also equals $2 \pi^{2} m_{e} e^{4} / h^{3} c$.) Not only that, but as mentioned earlier, Schrödinger's wave function that disclosed the fine structure constant or "barcode" of hydrogen $(137+)$ is also derived from Leibniz's and Euler's exponential growth function known as the harmonic power series based upon the natural logarithm. (Lest we forget: the fine structure constant is the zero-energy limit of the coupling charge that combines the strong and electroweak forces in the atom.) The exponential growth function is the simplest possible differential equation, the unique solution to $d y / d x=y ; y(0) 1$, and the most primitive (prime) derivative in which state and rate, form and function, symmetry and dynamism, being and 
becoming, structure and process, the integral and the derivative, the evolute and the involute, the "splice" and the "slice," and the squaring of a root and the extraction of a root are the same. It bears emphasizing that this function (and its constant multiples) is the only growth function with all these special characteristics. There is, of course, an intimate connection between primes and the natural logarithm through the prime number theorem, except that in order for the exponential growth function to use the natural $\log$ of 2.718 , it has to be the case that the first factorial in the exponential series, $1 / 0$ !, is equal to one, like the second factorial $1 / 1$ !. Otherwise, the sum of the series only adds up to $1.718 \ldots$ But how can this indeterminate expression be equivalent to a determinate unity without introducing an ad hoc fudge factor at the beginning of the factorials to paper over and finesse the gap? The answer is to be found in the indeterminacy of quantum mechanics. There has to be a quantum jump (a Fibonacci energizer bunny hop!) that truly bridges the gap between the indeterminate beginning and the subsequent definite series, and that is the dimensionless number $137+$, or Planck's constant, which is the basis of the quantum principle of indeterminate determinacy. The Planck bridge has gaps between its planks, which is the reason why it can join indeterminate zero with determinate one.

In a metaphorical manner of speaking, quantum mechanics was "conceived immaculately," on the basis of a pure, dimensionless, selfderived "virgin" number, Planck's constant 137+, which is also how the entire universe was "conceived out of a pure vacuum" in full accord with the principle of least action. Furthermore, as we will see, the number 137+ unites some, if not all, of the fundamental constants of the universe: action, light, heat, the electron charge, etc. Without this Goldilocks number, to put it bluntly, you and I would not be here. And the question arises, would anything?

Leibniz, of course, deemed this universe the "best" or optimal one of all possible universes. In his first formulation of the principle of stationary action (in a letter to a friend of Maupertuis, the latter of whom also formulated a version of this principle), Leibniz writes,

"The action is not as you thought, time should enter into it, it is as the product of mass by distance and time. I have noticed that, in the modifications [variations] due to motions, it usually becomes a maximum or a minimum. One could derive from that several statements of great import; it could help to determine the trajectories of bodies attracted by one or several others [Koenig, Acta Eruditorum, 1751]." 
In just these few short sentences the link was forever forged between the principle of least action (work) and the parameterizing triplet of mass $(M)$, distance $(L)$, and time $(T)$ that is the foundation of the physical sciences (least action, for example, equals $M L^{2} T$ ). (In its official emblem the American Physical Society symbolizes these three fundamental pillars with a brass weight, a standard ruler, and an oscillating pendulum, respectively.)

Newton's and Leibniz's calculus, exponential function, and binomial theorem all depend upon this initial leap from nothing to something (the amPhi-bian $\sqrt{ }-1$ ), or the pure number that transforms nothing into something. Without calculus physics is incomplete. Without the natural log calculus is incomplete. Without the Phi "Fibonacci energizer bunny" the natural log is incomplete. These would all be just castles in the air, without foundation. Phi is complete and self-contained because it is its own derivative, its own unity of zero and one (like its Greek symbol $\phi$ ) in a state of superposition, out of which derivatives like position and motion in calculus emerge. Without the calculus of variations and the principle of least action, the bridge between pure geometry and physics (the universe, too?) would collapse.

Another "exotic" bridge between mathematics and physics is the equivalence between light-speed (137+) and the so-called but mis-named "imaginary" square root of -1 in the best-known equation of all, $E=m c^{2}$. Since $c^{2}$ is equal to -1 seconds, $c$ itself equals the square root of -1 . According to relativity theory, the speed of light also follows the path of least time. As the time interval decreases, the angle between space and time approaches a right angle. When the time interval is exactly zero, the right angle is reached. It follows from all this that we owe the existence of the universe (and therefore ourselves) to this minus sign inside a square root. Something is easier than nothing; a complete vacuum is most difficult to attain and sustain.

Leibniz referred to this unusual "number" as the "amphibian between nothing and something" [Tobias Danzig, 1967]. He might just as well have said that it is the "am-Phi-bian" between them because this special equation of special relativity changes one dimension into another (time into space) just like the golden proportion because of Phi's uniquely combined arithmetical (additive) and geometrical (multiplicative) property. Leibniz, the co-discoverer of the principle of least action, along with Maupertuis and Euler, came within a (Fibonacci) "hare" of being the first quantum mechanic; both Planck and Einstein frequently acknowledged their debt to him. It almost goes without saying--be advised: be wise-quantize.

The 137+ of Planck's constant in Heisenberg's principle of indeterminacy is also a bridge between one dimension and another, since indeterminacy is responsible for the infinite zero point power of the quantum 
vacuum. Similarly, a vanishing zero point in the infinite distance produces a new dimension, like the three-dimensional effect in a two-dimensional Renaissance painting, and in calculus, a lower dimension is a zero (slice) in relation to a higher dimension (of infinitely spliced slices of the lower one). Planck's $h$ can equal infinite energy/zero time because $h=$ energy $\mathrm{x}$ time; when one or the other conjugate variable is zero the other one has to be infinite, in inverse proportion-they undo each other. In other words, zero wavelength is equal to infinite energy, like a black hole, and vice versa. In an infinitely curved black hole, time stops and mass is infinite. Astonishingly, this means that Planck's constant equals both $i$ and Phi, as well as $c$ or light-speed.

Consistent with this trans-dimensionality, a wavicle of light, like Phi, is both a whole and a part, a line and a point, as in the "duality" of projective geometry. At the speed of light, almost miraculously, an object becomes a cross-section of itself. If the first dimension is a cross-section of the second, and the second is a cross-section of the third, etc., what is the cross-section of a null line of light? It's a null-point that is its own cross-section, just as Phi is a cross-section of itself. In a word, both light and Phi are selfcontained, like a fractal. It also happens to be the case that when rays of light pass through two sheets of glass in contact with each other, there are multiple reflections, and the number of emergent rays is always a Fibonacci number, converging with an increase in rays onto Phi. This means that because of alpha, light, like hydrogen, is related to both $137 \mathrm{~s}$ - that of Phi and the charge of the electron squared. Since $i$ cannot have any roots, it, too, is its own cross-section or a 90-degree angle between horizontal and vertical lines, one dimension and another. (Lest we forget: the cosine of a 90-degree angle is zero, and two dimensions are 90 degrees out of phase.) Just as the rotation of the 90-degree angle twists the two forces of electricity and magnetism into one force (Clerk Maxwell's electromagnetism), like the Möbius strip, so does it combine line and plane, time and space, position and momentum, etc., into unities. One dimension is at a right angle to another, time is at a right angle to space, and position is at a right angle to momentum. Such is the rectification (justice, or "putting things to rights") of some very important fundamental constants and the entire universe.

\section{Summation over histories (time) to the summit}

Whoever reflects on this rectilinear answer to the deep cosmological question of existence will no doubt be reminded of Feynman's updated version of the principles of least time and action as the sum over possible histories to a limit. For him, the sum over all the possible pathways of light cancel out to the integral (limit) of least time (137+). By means of the Lagrangian calculus of variations, also, least action is the trajectory (path) 
that minimizes the action. According to this calculus, to be (a universe or anything else) is to be the value of a limit of a sum. (In connection with this, it is worth mentioning the mathematical logician W.V.O. Quine's insightful epigram: “To be is to be the value of a bound variable.") Consequently, a calculation of variations over the possible histories of the universe (possible universes) might disclose that they cancel each other out only to leave the singular limit of $137+$ as the optimal, most selfconsistent limit. Support for this conclusion comes from Phi and the exponential growth function which are the only self-consistent functions because they are self-derived, i.e., the only functions where "we can have our cake and eat it, too." (The cycloid of the pendulum swing also has this extraordinary property, but the evolute of a cycloid is another cycloid, and not the same one.)

This same spark of light with its fuse and fusion is what breathes life into the natural log and enables it to grow. Similarly, it's the fire in the exponential equation that enables the universe to inflate or explode! "The spreading light-wave [137] extends space," remarked cosmologist E. A. Milne. Since there is nothing, no space or time, for the universe (or the multiverse) to expand into, by definition, any further spacetime has to be produced out of absolutely nothing, exactly like the beginning of the universe. Least action and the quantum of action are responsible for the overall creation and expansion of the universe. Even if there is an everlasting universe or an expanding multiverse, with "universes" expanding into one another, like bubbles, the question has to arise as to what they, as a collection, and what the last universe, are all expanding into. (There is a similar push-back question, why is there this particular multiverse or set of universes rather than some other?) Unlike bubbles that are expanding into some already-existing outer space, bubbleuniverses as a whole have to be expanding into space that does not yet exist. This is something like a veritable creation of something out of nothing or quantum jump. Otherwise the logical fallacy of composition is committed, i.e., the mistake of attributing a quality, in this case an already existing outside space, to the whole that properly belongs only to the parts.

Interestingly, as our bubble universe inflates, it cools down, producing the cosmic background radiation that has a dampened, pendulum-like oscillation in accordance with the harmonic series of the natural logarithm [W. Hu and M. White, 2004]! The 1/0! factorial at the beginning of the series equals the number one because of the bridge of stationary action, just as many, if not all, fundamental constants equal one because of alpha 137 , as we will see.

So, what is there "before" or "beyond" the expanding universe or multiverse that is not spatio-temporal? "Before" and "beyond" position (not to be taken to mean spatio-temporal priority) there was superposition or the 
qubit, like the superposed symbol for Phi, $\phi$, which is both zero and one: $(0,1)$ is the Hamiltonian pair of numbers for $i$, the $\sqrt{ }-1$, and the quantum (order-in-chaos) jump from potentiality to actuality. The least amount of action is self-referral and self-derived, like Phi and the circle. For this reason, action is the self-referral curvature of spacetime, and so is gravity! Because action and gravity are equivalent to the curvature of space-time, the prime number 137 is responsible for both quantum action and gravityrelativity being equal to one. In accordance with the astonishing quantumelectrodynamic equation, $h c / 2 \pi e^{2}=137+$, the equation $\sqrt{ } h c / 2 \pi G$ is the greatest optimal way that these three constants of action, light, and gravity can be combined to get mass or space-time curvature.

Optimization appears not only in physics and chemistry but also in biology (Newton's laws of motion minimize action). For example: the proportion of white to grey matter in animal and human brains minimizes communication delay in the neurons; the firing of brain neurons follows the all-or-nothing binary system, coordinated by the minimizing principle; the body locomotion of animals optimizes travel by minimizing the use of energy so as to maintain the right ratio between the distance covered and the expenditure of energy. Living organisms develop in such a way so as to minimize the amount of energy necessary to distribute food, water, heat, and air-the scale of the four classical elements that led to the discovery of calculus and the derivatives (dimensions) by Leibniz and Newton ("earth" is position, "water" is uniform motion, "fire" is acceleration, "air" is force). This means that if something is not in proportion, economical, or scaleable, it's not saleable. In other words, be wise-digitize.

The employment of the calculus of variations is like a cost-benefit, risk-result analysis of possible universes. Leibniz's calculus is in part the inspiration behind George Dantzig's ingenious “simplex method" and its algorithm which are used worldwide today for deciding how to maximize results in the most efficient way. Dantzig's simplex algorithm is also based upon Descartes's and Euler's characteristic equation, Faces + Vertices Edges $=2$, since optimal value can be reached by following the edge where the target function descends the quickest (steepest), like the 90-degree angle. Ludwig Schlafli later generalized this "characteristic" to F + V - E Compartments $=0$, from point to volume, that produces a full three dimensions and beyond. Energy efficiency means moving three-dimensional mass the greatest distance in the shortest amount of time, and the golden ratio is the most effective means of accomplishing this task, which is why it is so ubiquitous in nature. Because the golden ratio is also the easiest, most efficient way to divide things up, cryptanalyst Alan Turing used it as his parsing theorem in all digital computation. In connection with this, it is worth emphasizing that since the four-dimensional cube has eight 
compartments and follows the Hamiltonian principle of least action, it is used for the optimal flow of information in parallel processing computers (W.D. Hillis, “The Connection Machine,” Scientific American, June 1988). For this reason there is also an eight-fold circuit in a cubic silicon computer chip.

Nature generally does everything in the most parsimonious, least work way possible. Because it has conservation laws, nature lives within its means - the golden mean (ratio-ration) in particular. That the least action principle lies hidden inside the golden ratio should not come as a complete surprise to us. Why? The arrangement of plant leaves in accord with the golden proportion angle of 137.5 degrees (called phyllotaxis) gives maximum exposure to water, light, and air (the classical elements again). The petals and florets of flowers (like roses and sunflowers) also are arranged according to the golden spiral so as to produce the most efficient, least wasteful, packing of space. Experiments with humans show that the eye is able to scan and enjoy the size of an object $(L)$ with the greatest ease $(M)$ in the least amount of time $(T)$ when the object perceived is in the Phi proportions. This is in accord with the ubiquitous fourfold (cubic) symmetry principle of nature, "short and slow should balance long and fast." That the longer horizontal dimension is scanned faster by our two eyes complements the shorter vertical dimension of slower visual speed. For this reason, sheets of paper, playing cards, credit cards, windows, doors, and even entire buildings (like the United Nations building in New York), etc., are designed to be as close to, if not exactly the same as, this famous ratio. "Shapes that resemble the golden ratio facilitate the scanning of images and their transmission through vision organs to the brain” (Design in Nature, Adrian Bejan and J. Peder Zane, page 233). This means that there is no fundamental distinction between mathematics, physics, art, and nature.

Following these two authors, we can maintain that "In the beginning was the [golden] proportion...," where fast and long meet slow and short, and the harmonic musical major meets the musical minor. It is the nondimensional nexus or bond between dimensions where it is indeed true that "fast into slow dives down below and slow into fast takes the upward path." In other words, the creation of the universe was as easy as $\mathrm{P}(h) \mathrm{i}$. (Or is it $\mathrm{P}(i)$ ?) This number is the boundary-condition or starting point (alpha) of the universe. The square root of minus one $(i)$, by the way, is equal to the square root of $e^{\pi i}$, according to Euler's "remarkable formula," $e^{\pi i}=-1$, a formula that upon being seen makes a person positively "pi-eyed." This formula is the foundation of the dimensions/derivatives in calculus and is a function of Euler's stunning equation for the "alphabetical" sine-cosine periodic motion: $e^{i x}=\cos x+i \sin x$. Furthermore, as Feynman discovered, $e^{\pi i}=1 /$ Phi - Phi.

Can there also be a connection between Phi and prime numbers? 
Since even the prime number "building blocks" of mathematics are grounded in the decimal system, and the decimal system is rooted in the $1,3,7, \ldots$ progression, there has to be an intimate connection between primes and these three numbers. And indeed there is: the place marker in the Fibonacci sequence of a Fibonacci number is a prime number! Moreover, excluding 2 and 5, all prime numbers end in 1, 3, 7, and 9 (think the "nine-test" of casting out nines and digital summation which is the test pattern for primes, using E. A. Midy's theorem based upon 1/7 or .142857 that transforms the decimal system into the binary code, as previously shown in the digital conversion chart). “...the least nonnegative prime residue system modulo 10 consists of the integers 1, 3, 7 and 9...” [Schroeder, 1984].

It is also worth mentioning in connection with the nine-test that any pair of twin primes, together with the composite number squeezed in between them, always digitally sum up to the number nine, e.g, $5+6+7=$ $9,11+12+13=9,17+18+19=9,179+180+181=9,191+192+193$ $=9,251+252+253=9$, etc. This is because any total sum of the three numbers in a twin-prime set is a multiple of the number nine. Such in part is the "dicemal" code of the prime numbers.

The English word “dice” comes from “dike,” in Greek, which means "justice," as in the title of Leibniz's only big book published in his lifetime, Theodice, "the Dice of God" [E. M. Huggard, 1966]. The primes are being justified, rectified, or put to "rights," as was first conjectured by Gauss using the prime number theorem and then hypothesized by Riemann in terms of the harmonic zeta function that is the "pre-established harmony" between primes and the non-trivial zeta zeros. (A bridge between the primes and the number $137+$ is an integral part of my published [novel] proof of the Riemann Hypothesis. Primes and zeta zeros are both quantized because of the Euler-Fourier-Riemann harmonic series wavefunction. See "Summa characteristica and the Riemann hypothesis: scaling Riemann's mountain,” the Journal of Interdisciplinary Mathematics, vol. 11, no. 6 [Dec. 2008], and "Generalizing Riemann: from the $L$-functions to the Birch/Swinnerton-Dyer conjecture," the Journal of Interdisciplinary Mathematics, vol. 13, no. 5 [Oct. 2010].)

\section{Why 137? (According to physicist Max Born, “... the number 137 is the dominating factor of all natural phenomena” [Max Born, 1932].}

According to quantum theory, light and matter are both particles and waves, or superposed wavicles, which, for Richard Feynman, are the "general mystery" of quantum physics [Richard Feynman, 1965]. It was Einstein's happy discovery that these two characteristics of light are bridged by Planck's constant of action. Since this discovery is so fundamental to quantum mechanics, it cannot be highlighted enough: without Planck's constant that combines particles and waves, momentum and position, energy 
and time, and many other conjugate pairs as well, quantum physics would collapse like a house of cards. (It is worthy of note that Planck's constant is the most ubiquitous number in quantum mechanics.) And what is the dimensionless number/ratio of light and Planck's constant that lies at the basis of all objects, not to mention that of the first element and its quantum electronic history pathways? It is, of course, Feynman's "mystery number" $137+$ that "all good theoretical physicists put up on their wall and worry about” [Richard Feynman, 1985].

As mentioned in the introduction, 137+ is the dimensionless number not only of hydrogen but of Einstein's constant of light, Planck's constant of action, and Feynman's charge of the electron, according to the quantum mechanical relativistic formula, $h c / 2$ pi $e^{2}$, known as alpha ${ }^{-1}$, the first letter of the Greek alpha-bet. (Should the letter $\alpha$ also stand for action?) It also appears in the Compton length which is 137 times smaller than the hydrogen atom. Because an electron in a hydrogen atom has a velocity $v=\alpha c$, it is therefore equal to light-speed divided by 137. Moreover, significantly, this number is the basis of Newton's constant of gravity, Boltzmann's constant of temperature in thermodynamics, Boltzmann-Maxwell's parameter of gas, and Coulomb's constant force of the atom whenever Max Planck's (and George Armstrong Stoney's) natural units are used to take the measurement of mass $(M)$, length $(L)$, time $(T)$ and temperature $(I)$. It cannot be emphasized enough that this is the number that makes many, if not all, fundamental constants equal to one.

The above-mentioned major fundamental constants in nature equal the number one (!), using these natural units, because the elementary charge of both the electron and the proton equals the square root of 137+ [Roger Penrose, 2004]. So, alpha ${ }^{-1}=G=h=c=k=i=$ epsilon $_{o}=1$, when $e=$ the square root of $137+$, making $137+$ the clasp between all the jewels in this stunning necklace, or the number one (1) number. When all of these constants except one of them equals one, the remaining one has to equal 137+. "What's it all about, Alfie?" It's all about alpha or "all-phi" (137+). This special ratio is the only way in which all of these constants can be combined into a unity, which goes a long way toward explaining "the unreasonable effectiveness of mathematics in relation to the physical sciences" [Eugene P. Wigner, 1960]. As incredible as it may seem, "Planck's units are the only combinations of these constants which can be formed with the dimensions of mass, length, time, and temperature" [John Barrow, 2002].

In the beginning was alpha, out of which leaped hydrogen, the first element, along with light $(c)$, heat $(k)$, and even gravity $(G)$, the first of the four fundamental forces--like a white (Fibonacci) rabbit out of a black hat (rabbit hole). According to the alpha equation, $h c / 2 \mathrm{pi} e^{2}$, the lower limit of 
all actions (Planck's quantum constant) and the upper limit of all speeds (Einstein's constant of relativity) are neatly conjoined through the mediation of the electron charge, which is the square root of 137+. It's even the case that a rotating black (rabbit) hole, as discovered by physicist Paul Davies, flips from a negative to a positive specific heat when the square of its mass divided by the square of its spin parameter is equal to-would you believe? — the golden ratio [Marcus Chown, 2002]. (More on black holes in a moment.) Alpha 137 is not only present at the beginning of the universe, it is present throughout its entirety, from top to bottom. When $137+$ is combined with two other fundamental constants-1/1836, which is the ratio of the electron-to-proton mass, and Newton's gravitational constant-these three constants scale (ration, temper) the sizes of everything in the universe from the smallest sub-atomic particles to the largest super-superclusters of galaxies. The whole universe is "graded" on the logarithmic growth curve, and as such it easily passes the "Goldilocks" test (the universe in a logarithmic seashell!). "Any percent or so change one way in [alpha] will cause all stars to be red stars; and a comparable change the other way will make all stars be blue stars... In neither case will any star like the sun be possible... Man could never come into being in such a universe,” writes the legendary physicist-cosmologist John Wheeler [C. W. Misner, K. S. Thorne, J. A. Wheeler, 1971, and J. D. Barrow and F. J. Tipler, 1996].

Quantum physicist Werner Heisenberg once remarked that when this special number/ratio of hydrogen is understood, the mysteries of physics will “shrivel up" [Leon Lederman, with Dick Teresi, 1993]. The Nobel prizewinning physicist Victor Weisskopf is supposed to have remarked, "When hydrogen is understood, we will understand all of physics." When asked if he really said this, he replied, “No, but I wish I had” [Daniel Kleppner, 1999; John S. Rigden, 2002].

Since the pure golden number/ratio has both the deepest depth and the widest span of inclusion in nature through the constants of light (the "large") and stationary action (the "small"), as well as in the scale (of the universe) in between, it has enormous explanatory power. "A theory is more impressive the greater is the simplicity of its premise, the more different are the kinds of things it relates, and the more extended its range of applicability”--Albert Einstein [Paul A. Schilpp, 1943].

\section{The stroboscopic spectrum of the universe}

All of this evidence indicates that alpha $137+$ is the basis or boundary condition of the "pre-established harmony" at the prime "atomic" level of mathematics, physics, and cosmology, for the reason that the essences of things are numbers, especially primes. Without prime numbers as foundational building blocks and their cornerstone--the "prime” prime 137+- 
-mathematics and physics would crumble.

"There is a deep connection between the symmetry principles and the conservation laws, but that connection requires that the minimum principle [of least action] be assumed.... In the last analysis the connection of symmetry laws to conservation laws comes from quantum mechanics," wrote Feynman [Richard Feynman, 1965].

To highlight this important point, let's sum up the exquisite chain of reasoning that leads to this summit (integral). The Principle of Indeterminacy hinges on Planck's constant which in turn establishes the Planck scale where the Planck natural units create the parameters of $M, L, T$, and $I$ that characterize the entire universe and unify the fundamental constants. Without indeterminacy and Planck's constant of least action, the bottom drops right out of the physical sciences and plunges them into the abyss of infinity.

Here is how superstring theorist Anthony Zee explains it: "The principle of action states that [a] particle actually follows the history with the smallest action.... Action is equal to the quantity, kinetic energy minus potential energy, summed over the history.

"The [least] action principle turns out to be universally applicable in physics. All physical theories established since Newton may be formulated in terms of an action. The action formulation is also elegantly concise.... The reader should understand that the entire physical world is described by one single action.... Our search for physical understanding boils down to determining one formula.... The action principle allows us to make precise the phrase, 'structure of physical reality'.... The action, in short, embodies the structure of physical reality" [Anthony Zee, 1999].

Still another quantum physicist, Leonard Susskind, emphasizes least action this way:

"The principle of least action-really the principle of stationary action-is the most compact form of the classical laws of physics. This simple rule (it can be written in a single line) summarizes everything! Not only the principles of classical mechanics, but electromagnetism, quantum mechanics, everything known about chemistry-right down to the ultimate known constituents of matter, elementary particles.... The [least] action is an integral, but, as always, an integral is the limit of a sum.... All the parameters... and all the equations of motion are packaged in a single function-the Lagrangian (kinetic energy minus potential energy, the integral of the Lagrangian)" [Leonard Susskind and George Hrabovsky, 2013]

In yet another place, with regard to Planck's natural units for mass, length, and time, Susskind makes the important claim that

"Many physicists think that there is a sense in which the Planck 
length is the smallest distance that can ever be resolved. Likewise, the Planck time may be the shortest interval of time. Let's not leave out the Planck mass.... The Planck mass is neither the smallest nor the largest possible mass of a black hole.... a Planck mass black hole would be about one Planck length in size and it would last for about one Planck unit of time before exploding into photons and other debris.... Recall that we estimated the vacuum energy density due to virtual particles. Not surprisingly, the answer translates to about one Planck mass per cubic Planck length. In other words, the unity of energy density that I defined as one Unit [his capitalization] was nothing but the natural Planck unit of energy density" [Susskind, 2006].

This strengthens the view that, with this unity of mass, length, and time in terms of Planck's natural units, the universe has hit a black "hole-inone." Furthermore, in connection with the physical constants Susskind asserts that

"If we reached into a bag of random numbers and pulled out generic values for the constants of nature, neither a small cosmological constant nor a suitable period of Inflation would be likely outcomes. Both require an enormous amount of fine-tuning.... What if the fine structure constant [1/137] were bigger, say about one? This would create several disasters, one of which would endanger the nucleus [of an atom].... Why is the fine structure constant small? No one knows, but if it were big, there would be no one to ask the question" [Susskind, 2006].

Here and elsewhere [see references] I have argued that an answer to this (fine structure, fine-tuned) question is to be found in the self-referral characteristic of the quantum mechanical (Planck) principle of least action and its corresponding self-derived geometrical ratio, the fairest division of them all, the golden ratio. (It will be remembered that Paul Davies found the golden ratio in black holes.) Support for this thesis has been electronically posted in a similar set of claims made by California physicist A. Garrett Lisi in "E8, An Exceptionally Simple Theory of Everything," that is based upon the recently deciphered Lie E8 symmetry group. What does this "exceptional" group have to do with stationary action and the golden proportion? Quite remarkably, according to a paper by Radu Coldea of Oxford University published in January, 2010: when the spins of superchilled cobalt niobate crystals were precisely magnetized into a state of quantum uncertainty, a fractal pattern appeared in which the interaction between spins made a series (scale) of resonant notes--the relationship between the first two notes being the golden ratio of 1.618! Coldea is convinced that the presence of the golden proportion is not an accident. "It reflects a beautiful property of the quantum system-a hidden symmetry. Actually quite a special one called E8 by mathematicians, and this is its first 
observation in a material [medium]” [R. Coldea et al., 2010].

Lest it be forgotten, the fine structure constant of the prime element hydrogen is $137+$, and the pathways of its electrons follow the Fibonacci sequence of numbers exactly. To understand hydrogen is to understand much, if not all, of physics. All of this seems almost too good to be true. How can this be?

\section{Conclusion (summing up): a uni-Phi-ed theory (the Tau) of science}

Like Leibniz, Einstein wondered whether a God had any choice in making the universe. Did a God choose this universe because of its harmony, or is there harmony because a God chose it? In a quantum, self-referral, participatory universe, this question is based upon a false disjunction, the black or white fallacy, like the dualism between wave and particle. Because of superposition and quantum logic (the logic of "both-and"), the two are not mutually exclusive.

This universe was "selected” (using Planck's natural units) because of its pre-established harmony, but the harmony presupposes the selfreferential Phi, etc., in the beginning, or else the harmony is incomplete. So, yes, there was a choice amongst possible universes because of the original indeterminacy in the pre-established harmony. But, no, there was no choice because the harmony is so beautiful and compelling. Both diversity (freedom) and unity (harmony) are present together in $\phi, i, h$, and $c$, like the golden mid-section between two sides of the same coin. (Spin a coin fast enough, and the two sides seem to "coincide.") Light (c or 137+), like everything in the universe, always follows the path of the beautiful (optimization), and beauty is in the Phi of the beholder, which means that beauty is both superpositionally invented and discovered. Like all great equations, proofs, and physical theories, beauty is both surprising and inevitable (as G. H. Hardy claimed).

Because opposites coincide, the separation between theory and fact, subject and object, the rational and the empirical, the ideal and the real, mathematics and physics breaks down. Speculation and experiment become superposed (like a thought-experiment); the same point that produces one produces the other. Because of superposition they are all tangled up. This means that the measure and the measured are not independent of each other. There is simply "bootstrap" self-referral self-measurement, self-action, selfintersection, self-derivation, self-representation, and self-containment-all of which are represented by the number 137+. Quantum mechanics basically threw everyone for a loop, because of this "strange loop" of self-action. In short, the measure without the measured is empty; the measured without the measure is blind.

We know that we have reached a fundamental, rock-bottom level 
whenever ostensible opposites become merged into an irreducible, selfderived, self-encroaching, self-supporting unity like Phi, wavicles, stationary action, spacetime, quanta of light, etc. At this juncture point no further differentials can be calculated, e.g., the difference between the product of position and momentum cannot be less than Planck's constant of 137+, and the self-derived golden ratio cannot be further reduced. Extracting square roots through calculus enables us to arrive at the square root of all roots, the root (origin) of all things or first principle, and put everything to "rights." By digging deeper and deeper we eventually reach the very foundation of self-referral measurement, which is as far down as the Fibonacci rabbit-hole goes. The primordial prime 137 is the universe's ace-in-the hole, its hole-inone.

This root number is not only the lowest common denominator or coupling constant between math and physics, it also links chemistry to biology since both begin with hydrogen, the mother of all the elements. Hydrogen bonds join together and shape the (top down) golden decagonal (digital) double helix form of DNA, as do the similarly supportive hydrogen bonds of water, the one non-negotiable prerequisite for life. If everything in the universe boils down to hydrogen and its quantum dualities, and all of these dualities boil down to a single constant, and everything is a combination of waves and particles based upon this same single constant-plus, this constant cannot be boiled down any further since it is self-derived and self-differentiated, the mother of all the constants - what else can the fundamental dimensionless number/ratio of the universe be? The differences among the differences have all been calculated down to zero in the golden number. (Phi's the limit.) This is where the rubber finally meets the road.

Moreover, the golden ratio is the only section that is created out of the self-interaction of the number one (the simplest continued fraction and the simplest nested or "radical" radical), the only number that is its own reciprocal when the number one is added to or subtracted from it $(1.618=.618+1)$, the only number and its reciprocal whose multiplication and difference are both equal to one, and the only number that is one less than its square. Phi is also the most irrational of all the irrational numbers, which is why it is the very last number, like $137+$ in quantum mechanics, to disappear before chaos (indeterminacy) sets in. In a word, it is the limit between order (stability) and chaos ( instability) in all fractal dynamic systems. (It is on the edge between determinacy and indeterminate chaos.) As if this were not enough, Phi is also the only division in which the "parts" remain one whole even in their separation, the only division that is a two-term proportion, the only proportion in which the addition and the multiplication of itself are equivalent, and, perhaps most significant of all, the only number that any two numbers converge to--all numbers, without exception, being the result of 
putting together powers of the golden section.

Because they are pure, fundamental, dimensionless numbers or ratios, both Planck's constant of action (137+) and the golden proportion (137.5 degrees), like the square root of minus one, are the connecting links between all the dimensions in mathematics and all the derivatives in physics and, last but not least, also help explain why there is something rather than nothing. To find an explanation is to find the middle term between one thing and another. In mathematics and physics the middle terms are 137+ and Phi, which are self-explanatory and self-justifying because they are self-derived. To sum it all up (to the limit): alpha ${ }^{-1}$ equals $137+$, making seven fundamental constants, $i=h=c=k=G=e^{2}=$ epsilon $_{o}$ equal to one because of Planck's natural units for mass, length, and time $(M, L, T)$ based upon the Planck scale, which here again is as far down as the (Fibonacci) rabbit hole can go. So, why does the glove of mathematics fit the hand of the physical sciences so well? It is because all of the glove's numbers, following the Fibonacci progression, converge to the golden section, and all the elements of physics and chemistry are based upon the prime element of hydrogen whose "fingers" or "digits" (the quantum histories) follow the Fibonacci progression toward the same golden ratio. Indeed, the segments of the fingers and hand all expand like a telescope in accord with the Fibonacci numbers so that when we make a fist the golden logarithmic spiral surprisingly appears. This is the (pure) gold standard, their original fit, the pre-established "arrival of the fit." Furthermore, as we saw earlier, the digital decimal code shares the same number of digits as the hands do, and the conversion of the digital code to the simple binary system sequentially follows the progression 1, 3, 7, etc., using the smallest, simplest, most fundamental cyclic number fraction, one divided by seven (seven is the pure “immaculate number" because it has no divisors and gives birth to itself).

Size 137+ is the perfect fit between the hand of physics and the glove of mathematics; one size (glove) fits all.

The consistent simplicity and simple consistency amongst all of these fundamental numbers, constants, and codes are no accident. Their symmetry and congeniality are the result of a common gene, the number $137+$, that, because of its unusual uniqueness (oneness) and self-referral irreducibility, is the generator, origin, and foundation of the fit between the "DNA" of mathematics, physics, chemistry, biology, and cosmology. Here indeed is the complete coalescence of mathematics and the physical sciences in terms of the number-ratio 137+, the super-formula summit, summa of all summation of actions, or summa mathematica/physica.

In the beginning was the word, and the word was alpha. 


\section{References:}

John Barrow, The Constants of Nature, New York, N.Y.: Pantheon Books, 2002, page 26 .

Max Born, “The Mysterious Number 137," Proceedings of the Indian Academy of Science, Sec. A. 2, 1932, page 547.

Marcus Chown, "Go figure. Why should nature have a favourite number?” New Scientist, December 21/28, 2002.

Radu Coldea et al., "Quantum Criticality in an Ising Chain: Experimental Evidence for Emergent E8 Symmetry,” Science, Jan. 8, 2010. (Contact: Prof. Alan Tennant at tennant@helmholtz-berlin.de). Public release: "Golden ratio discovered in a quantum world. Hidden symmetry observed for the first time in solid state matter":

http://www.eurekalert.org/pub_releases /2010-01/haog-grd0010510.php.

Tobias Danzig, Number, the Language of Science, New York, N.Y.: The Free Press, fourth edition, 1967, page 204.

Richard Feynman, The Character of Physical Law, Cambridge, MA.: The M.I.T. Press, 1965, pages 103-106.

Richard Feynman, The Character of Physical Law, Cambridge, MA.: The M.I.T. Press, 1965, page 130.

Richard Feynman, QED, The Strange Theory of Light and Matter, Princeton, N.J.: Princeton University Press, 1985, page 129.

Wayne Hu and Martin White, "Special Report on the Cosmic Symphony," Scientific American, Feb., 2004.

E. M. Huggard, trans., Leibniz’s Theodicy, New York, N.Y.: BobbsMerrill Co., 1966.

H. E. Huntley, The Divine Proportion, New York, N.Y.: Dover Publications, Inc., 1970, page 156.

Daniel Kleppner, “The Yin and Yang of Hydrogen,” Physics Today, 52(4) , page 11 (1999); doi: 10.1063/1.882619. Also, part of the quotation is to be found in John S. Rigden, Hydrogen, the Essential Element, Cambridge, MA.: Harvard University Press, 2002, page 253.

Robert Latta, ed., Leibniz's Monadology, Oxford: Oxford University Press, 1965, page 409. 
Leon Lederman, with Dick Teresi, The God Particle, New York, N.Y.: Delta, 1993, page 28.

Mario Livio, The Golden Ratio, the Story of Phi, the World's Most Astonishing Number, New York, N.Y.: Broadway Books, 2002, page 175.

C. W. Misner, K. S. Thorne, J. A. Wheeler, Gravitation, New York, N.Y.: W. H. Freeman and Co., 1971, page 1216, and J. D. Barrow and F. J. Tipler, The Anthropic Cosmological Principle, Oxford: Oxford University Press, 1996, page vii.

Paul J. Nahim, The Story of i, An Imaginary Tale, Princeton, N.J.:

Princeton University Press, 1998, page 67.

G. H. R. Parkinson, ed., Leibniz Philosophical Writings, London: Everyman’s Library, 1983, page 3.

Roger Penrose, The Road to Reality, New York, N.Y.: Alfred A. Knopf, 2004, pages $870 f$.

Paul A. Schilpp, ed., Albert Einstein, Philosopher/Scientist, in The Library of Living Philosophers, vol. 7, La Salle, IL.: Open Court Publishers, page 33.

Manfred R. Schroeder, Number Theory in Science and Communication, Berlin, New York: Springer-Verlag, 1984, page 35.

Leonard Susskind, The Cosmic Landscape, New York, N.Y.: Little, Brown and Co., 2006, Pages 87, 167 and 175.

Leonard Susskind and George Hrabovsky, The Theoretical Minimum, New York, N.Y.: Basic books, 2013, pages 105, 115, and 116.

Eugene P. Wigner, "The Unreasonable Effectiveness of Mathematics in the Natural Sciences," Communications on Pure and Applied Mathematics $13,1,1960$, pages 1-14.

Anthony Zee, Fearful Symmetry, Princeton, N.J.: Princeton University Press, 1999, pages 142 and 214. 